\title{
Serum phosphorylated neurofilament heavy-chain levels reflect phenotypic heterogeneity and are an independent predictor of survival in motor neuron disease
}

\author{
Yuri Matteo Falzone ${ }^{1,2} \cdot$ Teuta Domi $^{1,2} \cdot$ Federica Agosta $^{3,4} \cdot$ Laura Pozzi $^{1,2} \cdot$ Paride Schito $^{1,2} \cdot$ Raffaella Fazio $^{5}$. \\ Ubaldo Del Carro $^{6} \cdot$ Alessandra Barbieri $^{5,7} \cdot$ Mauro Comola $^{7} \cdot$ Letizia Leocani $^{3,7}$. Giancarlo Comi ${ }^{2} \cdot$ Paola Carrera $^{8}$. \\ Massimo Filippi ${ }^{2,3,4,5,6} \cdot$ Angelo Quattrini ${ }^{1,2} \cdot$ Nilo Riva $^{1,2,5,7}$
}

Received: 23 January 2020 / Revised: 10 April 2020 / Accepted: 13 April 2020 / Published online: 18 April 2020

(c) Springer-Verlag GmbH Germany, part of Springer Nature 2020

\begin{abstract}
To investigate the prognostic role and the major determinants of serum phosphorylated neurofilament heavy -chain $(\mathrm{pNfH})$ concentration across a large cohort of motor neuron disease (MND) phenotypes. Enzyme-linked immunosorbent assay (ELISA) was used to measure serum pNfH concentration in 219 MND patients consecutively enrolled in our tertiary MND clinic. A multifactorial analysis was carried out to investigate the major clinical determinants of serum pNfH. Kaplan-Meier survival curves and Cox regression analysis were performed to explore the prognostic value of serum pNfH. Serum $\mathrm{pNfH}$ levels were not homogenous among MND phenotypes; higher concentrations in pyramidal, bulbar, and classic phenotypes were observed. C9orf72-MND exhibited higher pNfH concentrations compared to non-C9orf72 MND. Multiple linear regression analysis revealed mean MEP/cMAP and disease progression rate as the two major predictors of serum $\mathrm{pNfH}$ levels $\left(R^{2}=0.188 ; p \leq 0.001\right)$. Kaplan-Meier curves showed a significant difference of survival among MND subgroups when divided into quartiles based on $\mathrm{pNfH}$ concentrations, log-rank $X^{2}=53.0, p \leq 0.0001$. Our study evidenced that higher serum $\mathrm{pNfH}$ concentration is a negative independent prognostic factor for survival. In Cox multivariate model, pNfH concentration showed the highest hazard ratio compared to the other factors influencing survival included in the analysis. pNfH differs among the MND phenotypes and is an independent prognostic factor for survival. This study provides supporting evidence of the role of $\mathrm{pNfH}$ as useful prognostic biomarker for MND patients. Neurofilament measurements should be considered in the future prognostic models and in clinical trials for biomarker-based stratification, and to evaluate treatment response.
\end{abstract}

Keywords Amyotrophic lateral sclerosis · ALS · FTD · C9orf72 · Biomarkers

Electronic supplementary material The online version of this article (https://doi.org/10.1007/s00415-020-09838-9) contains supplementary material, which is available to authorized users.

Nilo Riva

riva.nilo@hsr.it

1 Division of Neuroscience, Neuropathology Unit, San Raffaele Scientific Institute, via Olgettina 48, 20132 Milan, Italy

2 Institute of Experimental Neurology (INSPE), San Raffaele Scientific Institute, Milan, Italy

3 Vita-Salute San Raffaele University, Milan, Italy

4 Division of Neuroscience, Neuroimaging Research Unit, San Raffaele Scientific Institute, Milan, Italy

5 Neurology Unit, San Raffaele Scientific Institute, Milan, Italy
6 Neurophysiology Unit, San Raffaele Scientific Institute, Milan, Italy

7 Neurorehabilitation Unit, San Raffaele Scientific Institute, Milan, Italy

8 Division of Genetics and Cell Biology, Unit of Genomics for Human Disease Diagnosis, San Raffaele Scientific Institute, Milan, Italy 


\section{Introduction}

Motor neuron disease (MND) includes a heterogeneous group of relentless neurodegenerative disorders defined and characterized by the degeneration of motor neurons. Amyotrophic lateral sclerosis (ALS) is the most common and severe form of MND, affecting upper (UMN) and lower motor neurons (LMN) leading to respiratory failure and death within 3-5 years after symptoms onset [1,2]. Despite this stereotypical definition, MND clinical spectrum includes extremely heterogeneous phenotypes characterized by a varying involvement of UMN and LMN, site of onset, cognitive and genetic characteristics, resulting in a different rate of progression and prognosis [3-6].

While extensive studies have been performed to better characterize clinical, cognitive, genetic and neuroimaging biomarkers of progression, and many potential biochemical markers in both cerebrospinal fluid (CSF) and serum have been proposed, no diagnostic and prognostic biomarkers are currently available in clinical practice [7-13]. Phosphorylated neurofilament heavy chain $(\mathrm{pNfH})$ and the neurofilament light chain (NfL) are nowadays considered as the most promising candidate biomarkers for MND [14]. Both pNfH and NfL have been extensively studied across the neurological disorders and are considered to be reliable markers of acute and chronic neuronal injury [15]. The diagnostic role of neurofilaments has already been extensively explored. Previous studies provided evidence of a better diagnostic performance and higher sensitivity and specificity of pNfH when tested in the CSF compared to serum; however, a strong correlation between serum and CSF concentration has been reported [9, 16, 17]. Furthermore, $\mathrm{pNfH}$ correlated with disease severity and survival parameters but no studies have so far investigated serum $\mathrm{pNfH}$ as an independent predictor of survival [10, 17-20]. On the contrary, NfLs were demonstrated as independent predictors of survival in both CSF and serum [18, 21, 22]. Currently no biochemical prognostic biomarkers for assessing neuronal damage and disease progression across MND phenotypes are been explored. There is, however, an urgent need for a biochemical prognostic biomarker for MND to facilitate the estimation of the progression rate, survival, and patient's stratification [8, 23]. Definition of subtypes of MND is required to predict disease course and to correlate the phenotypes to the serum $\mathrm{pNfH}$ as a disease marker.

We performed a monocentric study in a large MND cohort of patients aimed at exploring serum $\mathrm{pNfH}$ across the different phenotypes, ascertaining the major determinants of the serum $\mathrm{pNfH}$ levels and assessing the prognostic role of the serum $\mathrm{pNfH}$ in MND patients.

\section{Materials and methods}

\section{Patient's selection and clinical data collection}

Two hundred and nineteen consecutive MND patients, referred to the ALS center of the San Raffaele Scientific Institute, Milan, were consecutively enrolled between December 2014 and January 2019 in accordance with the Awaji and revised cEl-Escorial criteria [24, 25]. Serum blood samples for $\mathrm{pNfH}$ assay were collected at the first evaluation at our center. At collection time, demographics and clinical history were recorded and neurological assessment was performed as follows: Medical Research Council (MRC) scale of 0 to 5 (12 muscles for each side; score 0-120 points) [26]; MRC progression rate $(\triangle \mathrm{MRC})$ calculated as [(120 - MRC score)/disease duration at the serum sample]; ALS Functional Rating Scale-revised (ALSFRS-R) was obtained and disease progression rate ( $\triangle$ ALSFRS-R) was calculated as (48 - ALSFRS-R)/disease duration); UMN burden was assessed with the UMN score, calculated by totaling the number of pathological UMN signs at examination (score 0-16) [26]. Patients were staged in agreement with King's clinical staging system and categorized, at the time of diagnosis, into eight different MND phenotypes, according to the previously published classification criteria and fulfilling the period of clinical observation required for a reliable phenotyping $[3,27]$.

Patients who underwent respiratory function assessment for clinical purposes were assessed with a standard spirometry recording forced vital capacity (FVC) expressed as a percentage of predicted volume and with arterial blood gas $(\mathrm{ABG})$ analysis recording partial oxygen $\left(\mathrm{PaO}_{2}\right)$ and carbon dioxide $\left(\mathrm{PaCO}_{2}\right)$ pressures. Height and weight were measured, and body mass index (BMI) calculated. A subset of patients underwent metabolic assessment to determine predicted and measured resting energy expenditure by indirect calorimetry and metabolic index (MI) calculated as predicted/measured resting energy expenditure.

Neuropsychological screening was performed with Edinburgh Cognitive and Behavioural ALS Screen (ECAS) [28]. A further neuropsychological evaluation as recommended by the Diagnostic Criteria for the Behavioural Variant of Frontotemporal Dementia and the ALSFTD Consensus Criteria was performed [29, 30]. MND patients were grouped in agreement with the ALS-FTD Consensus Criteria into five categories: frontotemporal dementia (FTD) (ALS-FTD); behavioral impairment (ALS-bi); cognitive impairment (ALS-ci); combined cognitive and behavioral impairment (ALS-cbi), which includes patients who fulfill criteria for both ALS-ci and ALS-bi; normal neuropsychological evaluation (ALSmotor). Only patients who underwent neuropsychological 
testing after publication of the ALS-FTD Consensus Criteria were considered [5].

Motor nerve conduction studies were routinely performed to determine bilateral median and common peroneal compound muscle action potential (cMAP) amplitude (peak to peak), and the mean cMAP at the four limbs was then calculated. Routine trans-cranial magnetic stimulation (TMS) was performed to measure motor-evoked potential (MEP) amplitude at the four limbs. MEP/cMAP amplitude ratio was calculated for each limb, expressed as a proportion of the cMAP elicited after peripheral nerve stimulation in the same target muscle (MEP/cMAP ratio) [31, 32]. Subsequently, mean MEP/cMAP at the four limbs was calculated. Only patients who underwent neurophysiological testing in a period between \pm 2 months from serum sample collection were considered and data were retrospectively retrieved from medical records.

Survival defined as time from serum sample to survival event defined as death/tracheostomy, and time to King's stage 4 defined as time from symptoms onset to significant feeding or respiratory failure were calculated. Additionally, survival time from symptoms onset to death/tracheostomy was calculated. Patients were followed up by periodical phone calls; survival status was last updated in October 2019. The Ethics Committee of the San Raffaele Scientific Institute of Milan approved the current study and all the participants gave written informed consent.

\section{Sample collection and pNfH assay}

Serum samples were processed within $1 \mathrm{~h}$ of blood collection and were stored at $-80{ }^{\circ} \mathrm{C}$ prior until analysis. $\mathrm{pNfH}$ serum levels were measured in duplicate blinded to disease status by commercial ELISA using human phosphorylated Neurofilament $\mathrm{H}$ antibody (Biovendor, RD191138300R, Brno, Czech Republic). Kits were used according to the manufacturers' instructions. Serum concentrations below the analytical sensitivity were nominated $23.5 \mathrm{pg} / \mathrm{ml}$. The mean intra-assay coefficient of variation was $6.8 \%$. The median serum $\mathrm{pNfH}$ concentration in an age- and gender-matched control cohort of 27 healthy subjects ( 11 women and 16 men; median age at venipuncture: 60.2 years, IQR $53.5-64.8$ ) was $28.5 \mathrm{pg} / \mathrm{ml}$ (IQR 23.5-81.2). DNA was available from all patients in the study and patients were screened for hexanucleotide expansion in $C 9$ orf 72 gene, to investigate the correlation of serum $\mathrm{pNfH}$ in the presence of the hexanucleotide expansion as previously reported in the CSF [33]. The $C 9$ orf 72 gene analysis was performed as previously described [34].

\section{Statistical methods}

Normality data distribution was assessed with the Shapiro-Wilk test. Continuous variables are reported with median and interquartile range (IQR) while categorical variables with number and relative frequencies. Mann-Whitney $U$ test was used to evaluate differences between two groups. Kruskal-Wallis test and Dunn post hoc comparison were performed to verify differences among groups at a significance level of 5\%. To investigate correlation between serum pNfH and clinical variables Pearson correlation analyses was carried out for continuous variables and Spearman correlation for ordinal variables. Subsequently, hierarchical multiple univariate linear regression analysis (stepwise procedure) was performed to underline which variables best explained the variance of serum pNfH. Kaplan-Meier (KM) univariate analysis was carried out to determine the effect of serum $\mathrm{pNfH}$ on survival from serum sample, time to King stage 4 and survival from symptom onset. log rank tests (Mantel-Cox) were used to test for differences among groups and, when more than two ordinal strata were used, the linear trend for factor level test was performed. Subsequently, multivariable analysis with Cox proportional hazards model (enter method) was performed to estimate the proportional hazard ratios of $\mathrm{pNfH}$ on survival and on time to King stage 4. Cox regressions were adjusted for known factors that negatively influence survival in ALS patients [14]. Serum pNfH concentration was set up as quartile groups as follows: first quartile $(23.5-40.1 \mathrm{pg} / \mathrm{ml})$; second quartile (40.2-174.3 pg/ml); third quartile (174.4-363.6 pg/ $\mathrm{ml})$; fourth quartile $(>363.6 \mathrm{pg} / \mathrm{ml})$. All statistical tests were performed using SPSS 22.0 software (Technologies, Inc., Chicago, IL, USA). $p$ value was set at $p<0.05$.

\section{Results}

A total of two hundred and nineteen (87 women and 132 men) MND patients were enrolled in the current study. Demographics and clinical characteristics of the MND patients are summarized in Table 1 . In our cohort, the median serum pNfH concentration was $174.3 \mathrm{pg} / \mathrm{ml}$ (IQR 40.1-363.3 pg/ml). Serum pNfH concentration was significantly different among MND phenotypes: higher levels were detected in pyramidal, bulbar, and classic phenotypes while flail arm, pure lower motor neuron (PLMN), and pure upper motor neuron (PUMN) phenotypes showed the lowest concentrations as shown in Fig. 1a and Table 2. Genetic analysis identified the $C 9$ orf 72 hexanucleotide repeat expansion in eighteen (8.2\%) patients of our cohort. C9orf72 MND patients exhibited significantly higher $\mathrm{pNfH}$ concentrations (median $403.3 \mathrm{pg} / \mathrm{ml}$, IQR 203.3-563.5 pg/ml) compared to non-C9orf72 MND patients (median $157.4 \mathrm{pg} / \mathrm{ml}$, IQR 
Table 1 Demographics and clinical characteristics of MND patients

\begin{tabular}{ll}
\hline Gender, M/F & $132 / 87(60.3 \% / 39.7 \%)$ \\
Age at venipuncture (years) & $64.0(57.0-71.0)$ \\
Diagnostic delay (months) & $9.0(6.0-15.0)$ \\
ALSFRS-R (points) & $37.0(32.0-42.0)$ \\
$\Delta$ ALSFRS-R (points/month) & $0.7(0.4-1.2)$ \\
MRC score (points) & $100.0(84.0-111.0)$ \\
$\Delta$ MRC (points/month) & $1.3(0.6-2.5)$ \\
UMNs (points) & $8.0(3.0-11.0)$ \\
ECAS ALS SPECIFIC (points) & $75.0(56.0-85.0)$ \\
Total ECAS score (points) & $99.0(79.0-112.0)$ \\
FVC (\%) & $83.5(58.3-99.0)$ \\
PO2 (mmHg) & $78.4(71.8-88.3)$ \\
PCo2 (mmHg) & $41.4(38.3-47.0)$ \\
Basal metabolic rate (\%) & $92.0(84.7-102.3)$ \\
BMI (ratio) & $24.1(22.0-26.1)$ \\
Mean MEP/cMAP & $0.2(0.1-0.3)$ \\
Mean cMAP four limbs & $6.0(3.2-8.2)$ \\
C9orf72 expansion (no/yes) & $201 / 18(91.8 \% / 8.2 \%)$ \\
Disease duration at venipuncture (months) & $14.0(9.0-24.0)$ \\
Serum pNfH (pg/ml) & $174.2(40.1-363.6)$ \\
\hline
\end{tabular}

Values shown are $n$, percentage (\%) or median (interquartile range)

$M$ male, $F$ female, $\triangle A L S F R S-R$ ALS Functional Rating Scale Progression Rate, $\triangle M R C$ Medical Research Council Scale Progression Rate, ECAS Edinburgh Cognitive and Behavioural ALS Screen, $U M N s$ Upper Motor Neuron score, $F V C$ forced vital capacity, BMI body mass index, MEP/cMAP motor evoked potential/compound muscle action potential, $C 9$ orf 72 chromosome 9 open reading frame $72, p N f H$ phosphorylated neurofilament heavy chain

33.3-334.6 pg/ml) (Fig. 1b). MND patients staged in King's 3 and 4 showed significantly higher pNfH concentrations compared to patients staged in King's 1 and 2 (Fig. 1c and Supplementary Table 1). One hundred and eighteen patients underwent a complete neuropsychological assessment and were categorized according to Strong criteria as shown in Table 2. No statistical differences were detected when MND patients were grouped by cognitive/behavioral phenotypes, age at symptoms onset and gender (Fig. 1d and Supplementary Fig. 1a, b).

In univariate analysis, $\mathrm{pNfH}$ concentration showed a moderate correlation with disease progression rate $(0.317$; $p \leq 0.001)$ and an inverse moderate correlation with mean $\mathrm{MEP} / \mathrm{cMAP}$ at the four limbs $(r=-0.342 ; p \leq 0.001)$. Moreover, $\mathrm{pNfH}$ concentration was weakly correlated with several MND clinical characteristics as UMNs, King's stage system, diagnostic delay, and disease duration at the serum sample (Supplementary Tables 2-5; Supplementary Fig. 1c, d).

In a subset of patients in which all the data were available $(n=129)$, a hierarchical multiple regression was carried out including variables that were significant at the univariate analysis. The full model of mean MEP/cMAP at the four limbs, disease progression rate, King's stage system, disease duration at venipuncture and diagnostic delay was statistically significant, $R^{2}=0.188 ; p \leq 0.001$. The significant variables included in the model were the mean MEP/cMAP at the four limbs and the disease progression rate. MEP/cMAP at the four limbs was the major determinant of serum $\mathrm{pNfH}$ concentration (Supplementary Table 6).

KM survival (defined as time from serum sample to death/tracheostomy) curves showed a significant difference of cumulative survivals between MND subgroups when divided by $\mathrm{pNfH}$ concentrations log-rank (Mantel-Cox) $X^{2}=53.0, p \leq 0.0001$, with higher serum $\mathrm{pNfH}$ concentrations related to shorter survival (Fig. 2). The median survival was fourth quartile 9.0 months (95\% CI 7.0-11.0 months), third quartile 23.0 months (95\% CI 18.6-27.4 months), second quartile 28.0 months (95\% CI 13.4-42.6) and first quartile 33.0 months (95\% CI 16.1-49.9 months). The negative effect of pNfH concentration was confirmed when stratifying Kaplan-Meier curves by age at onset and disease duration at serum sample (data not shown). Additionally, Kaplan-Meier curves showed a significant stratification when time to King's stage 4 was considered as event log-rank (Mantel-Cox) $X^{2}=68.1, p \leq 0.0001$ and similarly when survival was defined as time from symptoms onset to death/tracheostomy log-rank (Mantel-Cox) $X^{2}=64.3, p \leq 0.0001$ (Fig. 2, Supplementary Fig. 2 and Supplementary Tables 7-12).

Multivariate Cox regression model confirmed that serum $\mathrm{pNfH}$ concentration is independently associated with a reduced survival (defined as time from serum sample to death/tracheostomy) in MND. Patients with serum $\mathrm{pNfH}$ concentration higher than $363.6 \mathrm{pg} / \mathrm{ml}$ (fourth quartile) showed an increased proportional hazard ratio (HR) of 3.67 (95\% CI 1.96-6.90) when compared with the first quartile group (Table 3 ). This result was confirmed by defining survival as time from symptoms onset to death/tracheostomy with an HR 3.86 (95\% CI 2.05-7.28) (Supplementary table 13) and considering the variables as continuous (Supplementary tables 14 and 15). Similarly, Cox multivariate analysis confirmed that serum pNfH concentration is an independent negative prognostic factor for time to King's stage 4, HR 3.55 (95\% CI 1.97-6.37) (Table 3). All the variables included in the different models, HR and 95\% CI are reported in Table 3 and in Supplementary Tables 13-15.

\section{Discussion}

Serum and CSF neurofilaments are promising diagnostic and prognostic biomarkers in MND. In our large cohort of MND patients, well characterized by several clinical parameters, we found that serum $\mathrm{pNfH}$ concentration was not homogenous among the MND phenotypes. Moreover, we demonstrated serum $\mathrm{pNfH}$ to be an independent predictive factor of survival in MND patients. 

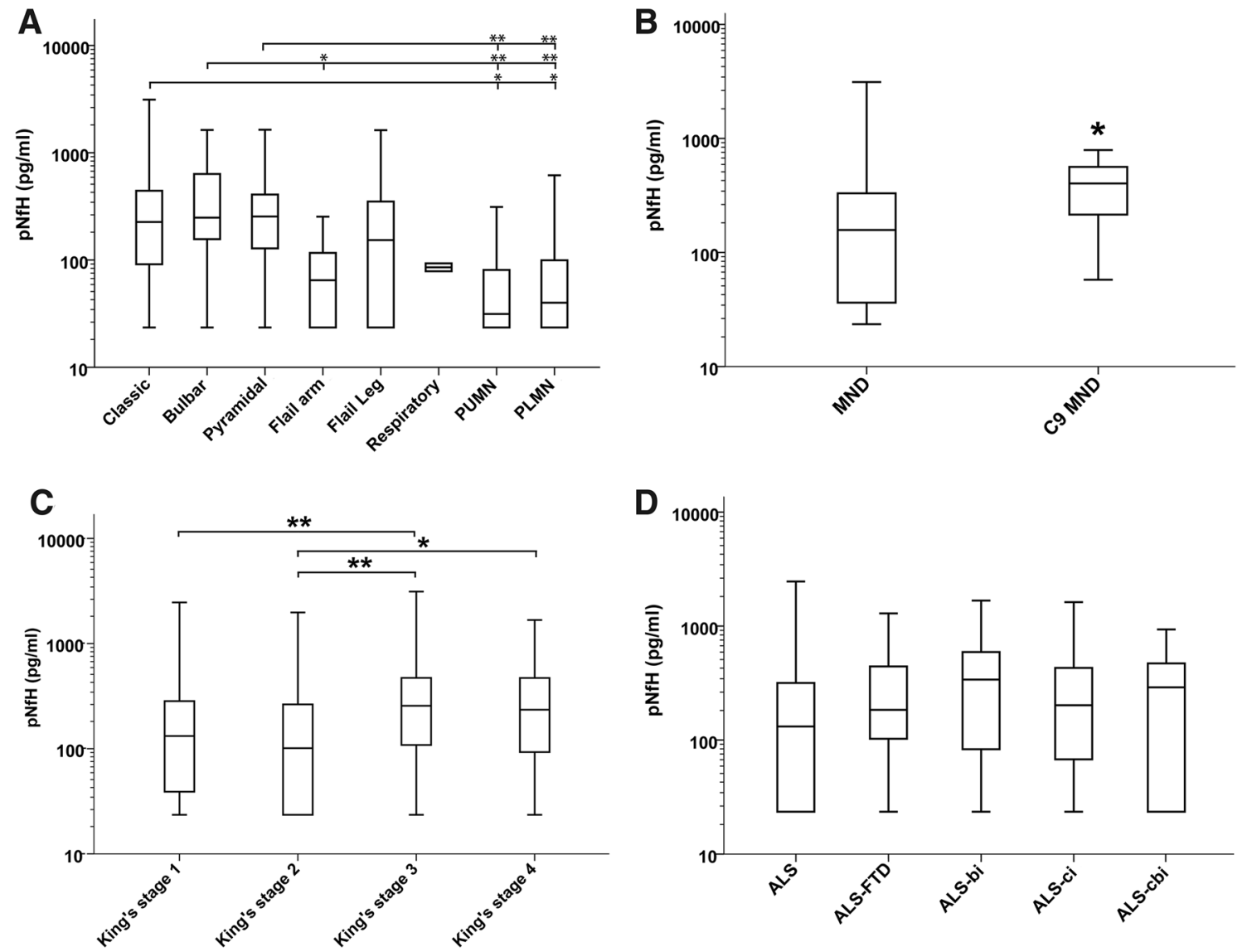

Fig. 1 Serum pNfH in MND patients. Boxplots showing pNfH concentrations among a MND phenotypes (overall groups comparison $p$ value $\leq 0.0001)$ grouped according to Chiò criteria [3]. b MND and C9MND patients, $\mathbf{c}$ MND patients staged according to King's stage system (overall groups comparison $p$ value $=0.001$ ) [27], d MND

Indeed, pyramidal, bulbar, and classic phenotypes exhibited higher $\mathrm{pNfH}$ levels compared to MND patients with a selective LMN or UMN involvement. The positive correlation between $\mathrm{pNfH}$ and the progression rate suggests that a faster degeneration of the motor system is one of the determinants of serum $\mathrm{pNfH}$ concentration, explaining the lower levels detected in slow progressive phenotypes as PUMN, PLMN, and FA. We found an inverse correlation between $\mathrm{pNfH}$ and MEP/cMAP suggesting that an elevated UMN burden might influence the serum $\mathrm{pNfH}$ concentration. Although the explanation is probably more complex, our results suggest that a rapid ongoing degeneration process of the UMN/corticospinal tract might be related to an increase of the serum $\mathrm{pNfH}$ concentration. However, MEP/cMAP does not selectively estimate UMN impairment and it might be influenced by both central and peripheral motor conductions; therefore, experimental neurophysiological methods such as short-interval intracortical inhibition (SICI) or advanced MRI investigations are needed to confirm this correlation. Consistent with

patients classified in agreement with Strong criteria (overall groups comparison $p$ value $=0.345$ ) [30]. $* p$ value $<0.05$; ** $p$ value $<0.01$. The median concentrations, $25 \%$ and $75 \%$ percentile and range values are given. pNfH levels are plotted on a 10-logarithmic scale

this hypothesis, previous studies reported that serum NfL levels were higher in ALS patients with a widespread UMN involvement and correlated with MRI measures of corticospinal tract degeneration, while NfL were lower in primary lateral sclerosis (PLS) patients [18, 35]. Notably, we observed significantly higher serum $\mathrm{pNfH}$ levels in C9orf72 MND patients compared to non-C9orf72 MND, in line with a previous study, which detected higher CSF pNfH levels in C9orf72 MND carriers [33]. C9orf72 MND has a faster disease progression rate and shorter survival [6], reflecting a widespread CNS neurodegeneration and more severe brain atrophy even involving extra motor areas compared to non- C9orf72 MND [34]. We investigated serum $\mathrm{pNfH}$ levels among the cognitive phenotypes, classified according to the Strong criteria [30]. Although MND patients with a concurrence of cognitive dysfunction and/ or FTD showed higher median pNfH levels compared to MND patients with normal cognition and behavior, this difference did not reach a statistical significance, suggesting that extra-motor areas involvement may not be a major 
Table 2 The pNfH concentration in different MND motor and cognitive phenotypes

\begin{tabular}{lll}
\hline & Case number & $\mathrm{pNfH}(\mathrm{pg} / \mathrm{ml})$ \\
\hline $\begin{array}{l}\text { Motor phenotype } \\
\text { Classic }\end{array}$ & $82 / 219(37.4 \%)$ & $226.2(89.6-449.5)$ \\
Bulbar & $31 / 219(14.2 \%)$ & $248.2(153.0-651.6)$ \\
Pyramidal & $31 / 219(14.2 \%)$ & $254.3(108.4-407.6)$ \\
Flail arm & $10 / 219(4.6 \%)$ & $70.6(23.5-120.4)$ \\
Flail leg & $30 / 219(13.7 \%)$ & $153.4(23.5-351.1)$ \\
Respiratory & $2 / 219(0.9 \%)$ & $85.8(-)$ \\
PLMN & $23 / 219(10.5 \%)$ & $40.0(23.5-112.2)$ \\
PUMN & $10 / 219(4.6 \%)$ & $32.7(23.5-127.6)$ \\
Cognitive phenotype & & \\
ALS motor & $52 / 118(44.1 \%)$ & $131.6(23.5-318.7)$ \\
ALS-FTD & $21 / 118(17.8 \%)$ & $184.1(90.4-523.7)$ \\
ALS-bi & $11 / 118(9.3 \%)$ & $339.2(74.2-688.0)$ \\
ALS-ci & $24 / 118(20.3 \%)$ & $202.3(66.9-435.3)$ \\
ALS-cbi & $10 / 118(8.5 \%)$ & $292.3(23.5-586.8)$ \\
\hline
\end{tabular}

Median values and interquartile range (IQR) are given

pNfH phosphorylated heavy chain, PLMN pure lower motor neuron, $P U M N$ pure upper motor neuron, $A L S-F T D$ frontotemporal dementia (FTD), $A L S$ - $b i$ behavioral impairment, $A L S$-ci cognitive impairment, $A L S$-cbi combined cognitive and behavioral impairment

determinant of the serum pNfH concentration in MND patients. However, further studies are needed to confirm our result. In support of our data, a previous study has shown higher CSF pNfH levels in ALS-FTD compared with FTD patients without evidence of motor system involvement, suggesting that the motor system involvement is the major determinant of $\mathrm{pNfH}$ concentrations [33].

While serum and CSF pNfH concentrations were previously correlated with survival parameters in univariate analysis, our study showed that serum $\mathrm{pNfH}$ concentration is a negative independent prognostic factor for survival through an accurate multivariate analysis [19]. Indeed, patients with higher $\mathrm{pNfH}$ concentration showed a significantly shorter median survival. Serum pNfH concentration showed the highest hazard ratio compared to all the other factors influencing survival included in the Cox multivariate model. Additionally, we showed that serum $\mathrm{pNfH}$ concentration was also significant as independent negative factor to predict the time to reach King's stage 4, i.e., the time to reach feeding or respiratory failure, which requires specific clinical interventions. Patients in King's stages 3 and 4 showed higher serum pNfH concentration compared to patients in King's stages 1 and 2. These findings suggest that $\mathrm{pNfH}$ levels in serum indicate the spreading and the global rate of CNS involvement and may offer a quick and simple measure to assess and predict neuronal damage in MND. Our results extend the previous findings that both neurofilament subunits and other wet biomarkers should be thoroughly assessed to be included in future prognostic models [18, 22, 36].

A partial limitation to the current study is that our results concerning serum $\mathrm{pNfH}$ were not replicable in the CSF due to the lack of serum and CSF paired matched samples in our cohort. Although previous works showed higher sensitivity and specificity of $\mathrm{pNfH}$ in the CSF when tested for a diagnostic purpose, a strong correlation between serum and CSF pNfH has also been demonstrated

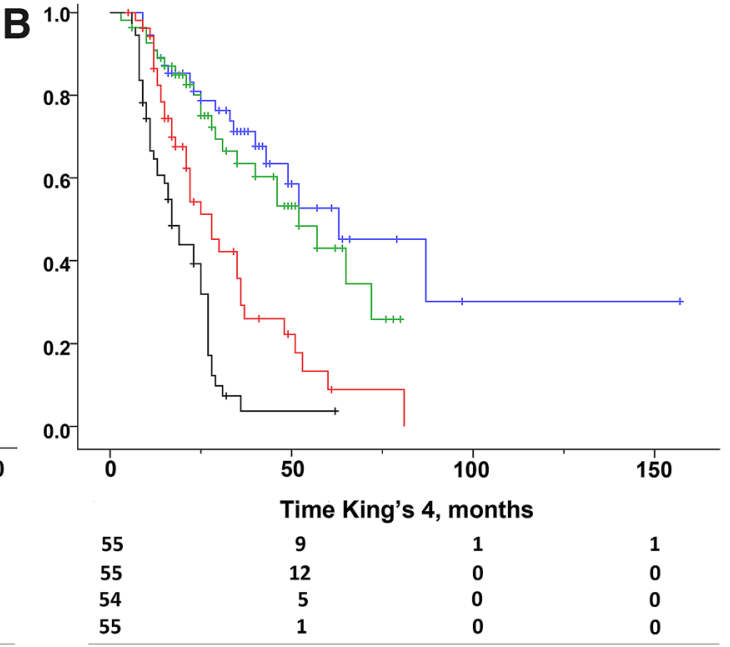

Fig. 2 Survival curves in MND patients a log-rank (Mantel-Cox) $X^{2}=53.0, p \leq 0.0001$ (event defined as time from serum sample to death/tracheostomy) b Kaplan-Meier time to King's stage 4 curves (event defined as time from symptoms onset to significant feeding or respiratory failure), log-rank (Mantel-Cox) $X^{2}=68.1, p \leq 0.0001$. MND patients were grouped according to quartile values; first quartile (blue line), second quartile (green line), third quartile (red line), and fourth quartile (black line) 
Table 3 Cox proportional hazards regression multivariate analysis on survival

\begin{tabular}{|c|c|c|c|c|}
\hline \multirow[t]{2}{*}{ Factor } & \multicolumn{2}{|c|}{$\begin{array}{l}\text { Survival (from serum sample to death or } \\
\text { tracheostomy) }\end{array}$} & \multicolumn{2}{|c|}{ Time to King's stage 4} \\
\hline & $\mathrm{HR}(95 \% \mathrm{CI})$ & $p$ value & HR $(95 \%$ CI $)$ & $p$ value \\
\hline $\begin{array}{l}\text { Serum pNfH concentration } \\
(\mathrm{pg} / \mathrm{ml})\end{array}$ & & $<0.001$ & & $<0.001$ \\
\hline $23.5-40.1$ & 1 & & 1 & \\
\hline $40.08-174.3$ & $1.27(0.66-2.43)$ & 0.480 & $1.41(0.75-2.63)$ & 0.288 \\
\hline $174.4-363.6$ & $1.55(0.83-2.89)$ & 0.167 & $2.45(1.37-4.39)$ & 0.003 \\
\hline$>363.6$ & $3.67(1.96-6.90)$ & $<0.001$ & $3.55(1.97-6.37)$ & $<0.001$ \\
\hline \multicolumn{5}{|c|}{ Disease duration at serum sample (months) } \\
\hline$\leq 14$ & 1 & & $1.74(1.09-2.77)$ & 0.021 \\
\hline$>14$ & $1.45(0.88-2.42)$ & 0.148 & 1 & \\
\hline \multicolumn{5}{|l|}{ Diagnostic delay (months) } \\
\hline$\leq 9$ & $1.04(0.66-1.64)$ & 0.874 & $1.23(0.82-1.84)$ & 0.321 \\
\hline$>9$ & 1 & & 1 & \\
\hline \multicolumn{5}{|c|}{ Progression rate (points/month) } \\
\hline$\leq 0.74$ & 1 & & 1 & \\
\hline$>0.74$ & $2.80(1.74-4.50)$ & $<0.001$ & $4.32(2.74-6.82)$ & $<0.001$ \\
\hline \multicolumn{5}{|l|}{ Dementia } \\
\hline No & 1 & & 1 & \\
\hline Yes & $1.61(0.92-2.82)$ & 0.095 & $1.18(0.70-1.99)$ & 0.542 \\
\hline \multicolumn{5}{|l|}{ C9orf72 expansion } \\
\hline No & 1 & & 1 & \\
\hline Yes & $1.30(0.62-2.69)$ & 0.488 & $1.16(0.59-2.27)$ & 0.662 \\
\hline \multicolumn{5}{|l|}{ Age at venipuncture (years) } \\
\hline $31-64$ & 1 & & 1 & \\
\hline$>64$ & $1.70(1.13-2.57)$ & 0.011 & $1.62(1.11-2.36)$ & 0.013 \\
\hline MND phenotype & & 0.058 & & 0.006 \\
\hline PUMN/PLMN/FA & 1 & & 1 & \\
\hline CL/PY/FL & $2.43(1.16-5.09)$ & 0.019 & $2.15(1.10-4.20)$ & 0.025 \\
\hline $\mathrm{B} / \mathrm{R}$ & $2.51(1.07-5.91)$ & $\mathbf{0 . 0 3 5}$ & $3.61(1.63-8.00)$ & 0.002 \\
\hline
\end{tabular}

Variables included in the model: $\mathrm{pNfH}$, phosphorylated neurofilament heavy chain subdivided into quartiles; disease duration at serum sample, diagnostic delay, age at onset, and progression rate divided according to the respective median values; presence of dementia no/yes; C9orf72, chromosome 9 open reading frame 72 hexanucleotide repeat expansion no/yes; MND phenotype, subdivided into three different groups: long survival group composed of pure upper motor neuron (PUMN), pure lower motor neuron (PLMN) and flail arm (FA); intermediate survival group composed of classic (CL), pyramidal (PY) and flail leg (FL); short survival group composed of bulbar (B) and respiratory (R)

$H R$ hazard ratio, $C I$ confidence interval in MND patients $[16,19,37,38]$. However, we aimed to assess the prognostic rather than the diagnostic role of serum pNfH. Furthermore, a prognostic blood-based biomarker would be preferable to a CSF biomarker to avoid an invasive practice such as the lumbar puncture. Nevertheless, we acknowledge that further independent confirmatory investigations are required to confirm our findings and to better define prognostic reference values. Furthermore, longitudinal studies will be essential to determine whether the $\mathrm{pNfH}$ levels increase, decrease or stay stable over time. Unfortunately, we are currently unable to raise additional experiments in the laboratory due to the lockdown related to the COVID-19 in Italy. Future studies will be essential to assess the variance of measurement of $\mathrm{pNfH}$ over the disease course.

In conclusion, our study shows that $\mathrm{pNfH}$ differs among MND phenotypes and is an independent prognostic factor for survival. This study provides evidence that supports the role of serum $\mathrm{pNfH}$ as useful prognostic biomarker for MND patients. Neurofilament measurements should be considered in future prognostic models and in clinical trials for biomarker-based stratification, and to evaluate treatment response. 
Author contributions YMF and TD performed the experiments; YMF, TD, PC, FA, MF, AQ, and NR contributed to analysis and interpretation of the data; YMF, PS, MC, AB, LL, UD, RF, and NR recruited the patients, performed the clinical evaluation, acquired clinical data, and recruited patients; TD, LP, and PC performed the genetic analysis; YMF, NR, and AQ drafted the manuscript; YMF, FA, MF, NR, and AQ conceptualized and designed the study; FA, MF, NR, and AQ obtained the funding; NR, AQ, GC, and FM supervised the study. All authors had full access to the data in the study, critically revised, and approved the final version of the manuscript.

Funding This study was partially supported by the Italian Ministry of Health (\#RF-2011-02351193).

\section{Compliance with ethical standards}

Conflicts of interest On behalf of all authors, the corresponding author states that there is no conflict of interest.

Patient consent Informed consent was obtained from the patients who participated in the study.

Ethics approval The study was approved by the appropriate ethics committee and has, therefore, been performed in accordance with the ethical standards laid down in the 1964 Declaration of Helsinki and its later amendments.

\section{References}

1. Riva N, Agosta F, Lunetta C, Filippi M, Quattrini A (2016) Recent advances in amyotrophic lateral sclerosis. J Neurol 263(6):12411254. https://doi.org/10.1007/s00415-016-8091-6

2. Hardiman O, Al-Chalabi A, Chio A, Corr EM, Logroscino G, Robberecht W, Shaw PJ, Simmons Z, van den Berg LH (2017) Amyotrophic lateral sclerosis. Nat Rev Dis Primers 3:17085

3. Chio A, Calvo A, Moglia C, Mazzini L, Mora G, Group Ps (2011) Phenotypic heterogeneity of amyotrophic lateral sclerosis: a population based study. J Neurol Neurosurg Psychiatry 82(7):740-746. https://doi.org/10.1136/jnnp.2010.235952

4. Montuschi A, Iazzolino B, Calvo A, Moglia C, Lopiano L, Restagno G, Brunetti M, Ossola I, Lo Presti A, Cammarosano S, Canosa A, Chio A (2015) Cognitive correlates in amyotrophic lateral sclerosis: a population-based study in Italy. J Neurol Neurosurg Psychiatry 86(2):168-173. https://doi.org/10.1136/jnnp2013-307223

5. McCann EP, Williams KL, Fifita JA, Tarr IS, O'Connor J, Rowe DB, Nicholson GA, Blair IP (2017) The genotype-phenotype landscape of familial amyotrophic lateral sclerosis in Australia. Clin Genet 92(3):259-266. https://doi.org/10.1111/cge.12973

6. Umoh ME, Fournier C, Li Y, Polak M, Shaw L, Landers JE, Hu W, Gearing M, Glass JD (2016) Comparative analysis of C9orf72 and sporadic disease in an ALS clinic population. Neurology 87(10):1024-1030. https://doi.org/10.1212/WNL.0000000000 003067

7. Knibb JA, Keren N, Kulka A, Leigh PN, Martin S, Shaw CE, Tsuda M, Al-Chalabi A (2016) A clinical tool for predicting survival in ALS. J Neurol Neurosurg Psychiatry 87(12):1361-1367. https://doi.org/10.1136/jnnp-2015-312908

8. Westeneng HJ, Debray TPA, Visser AE, van Eijk RPA, Rooney JPK, Calvo A, Martin S, McDermott CJ, Thompson AG, Pinto S, Kobeleva X, Rosenbohm A, Stubendorff B, Sommer H, Middelkoop BM, Dekker AM, van Vugt J, van Rheenen W, Vajda
A, Heverin M, Kazoka M, Hollinger H, Gromicho M, Korner S, Ringer TM, Rodiger A, Gunkel A, Shaw CE, Bredenoord AL, van Es MA, Corcia P, Couratier P, Weber M, Grosskreutz J, Ludolph AC, Petri S, de Carvalho M, Van Damme P, Talbot K, Turner MR, Shaw PJ, Al-Chalabi A, Chio A, Hardiman O, Moons KGM, Veldink JH, van den Berg LH (2018) Prognosis for patients with amyotrophic lateral sclerosis: development and validation of a personalised prediction model. Lancet Neurol 17(5):423-433. https://doi.org/10.1016/S1474-4422(18)30089-9

9. Li DW, Ren H, Jeromin A, Liu M, Shen D, Tai H, Ding Q, Li $X$, Cui L (2018) Diagnostic performance of neurofilaments in Chinese patients with amyotrophic lateral sclerosis: a prospective study. Front Neurol 9:726. https://doi.org/10.3389/fneur .2018 .00726

10. Steinacker P, Feneberg E, Weishaupt J, Brettschneider J, Tumani H, Andersen PM, von Arnim CA, Bohm S, Kassubek J, Kubisch C, Lule D, Muller HP, Muche R, Pinkhardt E, Oeckl P, Rosenbohm A, Anderl-Straub S, Volk AE, Weydt P, Ludolph AC, Otto $M$ (2016) Neurofilaments in the diagnosis of motoneuron diseases: a prospective study on 455 patients. J Neurol Neurosurg Psychiatry 87(1):12-20. https://doi.org/10.1136/jnnp-2015-31138 7

11. Taga A, Maragakis NJ (2018) Current and emerging ALS biomarkers: utility and potential in clinical trials. Expert Rev Neurother 18(11):871-886. https://doi.org/10.1080/14737 175.2018.1530987

12. Verber NS, Shepheard SR, Sassani M, McDonough HE, Moore SA, Alix JJP, Wilkinson ID, Jenkins TM, Shaw PJ (2019) Biomarkers in motor neuron disease: a state of the art review. Front Neurol 10:291. https://doi.org/10.3389/fneur.2019.00291

13. Conti A, Riva N, Pesca M, Iannaccone S, Cannistraci CV, Corbo M, Previtali SC, Quattrini A, Alessio M (2014) Increased expression of Myosin binding protein $\mathrm{H}$ in the skeletal muscle of amyotrophic lateral sclerosis patients. Biochim Biophys Acta 1842(1):99-106. https://doi.org/10.1016/j.bbadis.2013.10.013

14. Poesen K, Van Damme P (2018) Diagnostic and prognostic performance of neurofilaments in ALS. Front Neurol 9:1167. https ://doi.org/10.3389/fneur.2018.01167

15. Bacioglu M, Maia LF, Preische O, Schelle J, Apel A, Kaeser SA, Schweighauser M, Eninger T, Lambert M, Pilotto A, Shimshek DR, Neumann U, Kahle PJ, Staufenbiel M, Neumann M, Maetzler W, Kuhle J, Jucker M (2016) Neurofilament light chain in blood and CSF as marker of disease progression in mouse models and in neurodegenerative diseases. Neuron 91(2):494-496. https://doi. org/10.1016/j.neuron.2016.07.007

16. De Schaepdryver M, Jeromin A, Gille B, Claeys KG, Herbst V, Brix B, Van Damme P, Poesen K (2018) Comparison of elevated phosphorylated neurofilament heavy chains in serum and cerebrospinal fluid of patients with amyotrophic lateral sclerosis. J Neurol Neurosurg Psychiatry 89(4):367-373. https://doi.org/10.1136/ jnnp-2017-316605

17. Poesen K, De Schaepdryver M, Stubendorff B, Gille B, Muckova P, Wendler S, Prell T, Ringer TM, Rhode H, Stevens O, Claeys KG, Couwelier G, D'Hondt A, Lamaire N, Tilkin P, Van Reijen D, Gourmaud S, Fedtke N, Heiling B, Rumpel M, Rodiger A, Gunkel A, Witte OW, Paquet C, Vandenberghe R, Grosskreutz J, Van Damme P (2017) Neurofilament markers for ALS correlate with extent of upper and lower motor neuron disease. Neurology 88(24):2302-2309. https://doi.org/10.1212/WNL.0000000000 004029

18. Gille B, De Schaepdryver M, Goossens J, Dedeene L, De Vocht J, Oldoni E, Goris A, Van Den Bosch L, Depreitere B, Claeys KG, Tournoy J, Van Damme P, Poesen K (2019) Serum neurofilament light chain levels as a marker of upper motor neuron degeneration in patients with Amyotrophic Lateral Sclerosis. Neuropathol Appl Neurobiol 45(3):291-304. https://doi.org/10.1111/nan.12511 
19. Boylan KB, Glass JD, Crook JE, Yang C, Thomas CS, Desaro P, Johnston A, Overstreet K, Kelly C, Polak M, Shaw G (2013) Phosphorylated neurofilament heavy subunit (pNF-H) in peripheral blood and CSF as a potential prognostic biomarker in amyotrophic lateral sclerosis. J Neurol Neurosurg Psychiatry 84(4):467-472. https://doi.org/10.1136/jnnp-2012-303768s

20. Lu CH, Petzold A, Topping J, Allen K, Macdonald-Wallis C, Clarke J, Pearce N, Kuhle J, Giovannoni G, Fratta P, Sidle K, Fish M, Orrell R, Howard R, Greensmith L, Malaspina A (2015) Plasma neurofilament heavy chain levels and disease progression in amyotrophic lateral sclerosis: insights from a longitudinal study. J Neurol Neurosurg Psychiatry 86(5):565-573. https://doi. org/10.1136/jnnp-2014-307672

21. Gaiani A, Martinelli I, Bello L, Querin G, Puthenparampil M, Ruggero S, Toffanin E, Cagnin A, Briani C, Pegoraro E, Soraru G (2017) Diagnostic and prognostic biomarkers in amyotrophic lateral sclerosis: neurofilament light chain levels in definite subtypes of disease. JAMA Neurol 74(5):525-532. https://doi.org/10.1001/ jamaneurol.2016.5398

22. Lu CH, Macdonald-Wallis C, Gray E, Pearce N, Petzold A, Norgren N, Giovannoni G, Fratta P, Sidle K, Fish M, Orrell R, Howard R, Talbot K, Greensmith L, Kuhle J, Turner MR, Malaspina A (2015) Neurofilament light chain: a prognostic biomarker in amyotrophic lateral sclerosis. Neurology 84(22):2247-2257. https ://doi.org/10.1212/WNL.0000000000001642

23. Calvo A, Moglia C, Lunetta C, Marinou K, Ticozzi N, Ferrante GD, Scialo C, Soraru G, Trojsi F, Conte A, Falzone YM, Tortelli R, Russo M, Chio A, Sansone VA, Mora G, Silani V, Volanti P, Caponnetto C, Querin G, Monsurro MR, Sabatelli M, Riva N, Logroscino G, Messina S, Fini N, Mandrioli J (2017) Factors predicting survival in ALS: a multicenter Italian study. J Neurol 264(1):54-63. https://doi.org/10.1007/s00415-016-8313-y

24. Brooks BR, Miller RG, Swash M, Munsat TL (2000) El Escorial revisited: revised criteria for the diagnosis of amyotrophic lateral sclerosis. Amyotroph Lateral Scler Other Motor Neuron Disord 1(5):293-299

25. Schrooten M, Smetcoren C, Robberecht W, Van Damme P (2011) Benefit of the Awaji diagnostic algorithm for amyotrophic lateral sclerosis: a prospective study. Ann Neurol 70(1):79-83. https:// doi.org/10.1002/ana.22380

26. Riva N, Mora G, Soraru G, Lunetta C, Ferraro OE, Falzone Y, Leocani L, Fazio R, Comola M, Comi G (2019) Safety and efficacy of nabiximols on spasticity symptoms in patients with motor neuron disease (CANALS): a multicentre, double-blind, randomised, placebo-controlled, phase 2 trial. Lancet Neurol 18(2):155-164. https://doi.org/10.1016/S1474-4422(18)30406-X

27. Roche JC, Rojas-Garcia R, Scott KM, Scotton W, Ellis CE, Burman R, Wijesekera L, Turner MR, Leigh PN, Shaw CE, Al-Chalabi A (2012) A proposed staging system for amyotrophic lateral sclerosis. Brain 135(Pt 3):847-852. https://doi.org/10.1093/brain /awr351

28. Abrahams S, Newton J, Niven E, Foley J, Bak TH (2014) Screening for cognition and behaviour changes in ALS. Amyotroph Lateral Scler Frontotemporal Degener 15(1-2):9-14. https://doi. org/10.3109/21678421.2013.805784

29. Rascovsky K, Hodges JR, Knopman D, Mendez MF, Kramer JH, Neuhaus J, van Swieten JC, Seelaar H, Dopper EG, Onyike CU, Hillis AE, Josephs KA, Boeve BF, Kertesz A, Seeley WW, Rankin
KP, Johnson JK, Gorno-Tempini ML, Rosen H, Prioleau-Latham CE, Lee A, Kipps CM, Lillo P, Piguet O, Rohrer JD, Rossor MN, Warren JD, Fox NC, Galasko D, Salmon DP, Black SE, Mesulam M, Weintraub S, Dickerson BC, Diehl-Schmid J, Pasquier F, Deramecourt V, Lebert F, Pijnenburg Y, Chow TW, Manes F, Grafman J, Cappa SF, Freedman M, Grossman M, Miller BL (2011) Sensitivity of revised diagnostic criteria for the behavioural variant of frontotemporal dementia. Brain 134(Pt 9):2456-2477. https ://doi.org/10.1093/brain/awr179

30. Strong MJ, Abrahams S, Goldstein LH, Woolley S, McLaughlin P, Snowden J, Mioshi E, Roberts-South A, Benatar M, HortobaGyi T, Rosenfeld J, Silani V, Ince PG, Turner MR (2017) Amyotrophic lateral sclerosis - frontotemporal spectrum disorder (ALS-FTSD): revised diagnostic criteria. Amyotroph Lateral Scler Frontotemporal Degener 18(3-4):153-174. https://doi.org/10.1080/21678 421.2016.1267768

31. Riva N, Falini A, Inuggi A, Gonzalez-Rosa JJ, Amadio S, Cerri F, Fazio R, Del Carro U, Comola M, Comi G, Leocani L (2012) Cortical activation to voluntary movement in amyotrophic lateral sclerosis is related to corticospinal damage: electrophysiological evidence. Clin Neurophysiol 123(8):1586-1592. https://doi. org/10.1016/j.clinph.2011.12.013

32. de Carvalho M, Chio A, Dengler R, Hecht M, Weber M, Swash $M$ (2005) Neurophysiological measures in amyotrophic lateral sclerosis: markers of progression in clinical trials. Amyotroph Lateral Scler Other Motor Neuron Disord 6(1):17-28. https://doi. org/10.1080/14660820410020600

33. Gendron TF, Group CONS, Daughrity LM, Heckman MG, Diehl NN, Wuu J, Miller TM, Pastor P, Trojanowski JQ, Grossman M, Berry JD, Hu WT, Ratti A, Benatar M, Silani V, Glass JD, Floeter MK, Jeromin A, Boylan KB, Petrucelli L (2017) Phosphorylated neurofilament heavy chain: a biomarker of survival for C9ORF72associated amyotrophic lateral sclerosis. Ann. Neurol 82(1):139146. https://doi.org/10.1002/ana.24980

34. Agosta F, Ferraro PM, Riva N, Spinelli EG, Domi T, Carrera P, Copetti M, Falzone Y, Ferrari M, Lunetta C, Comi G, Falini A, Quattrini A, Filippi M (2017) Structural and functional brain signatures of C9orf72 in motor neuron disease. Neurobiol Aging 57:206-219. https://doi.org/10.1016/j.neurobiola ging.2017.05.024

35. Menke RA, Gray E, Lu CH, Kuhle J, Talbot K, Malaspina A, Turner MR (2015) CSF neurofilament light chain reflects corticospinal tract degeneration in ALS. Ann Clin Transl Neurol 2(7):748-755. https://doi.org/10.1002/acn3.212

36. Thouvenot E, Demattei C, Lehmann S, Maceski-Maleska A, Hirtz C, Juntas-Morales R, Pageot N, Esselin F, Alphandery S, Vincent T, Camu W (2019) Serum neurofilament light chain at time of diagnosis is an independent prognostic factor of survival in amyotrophic lateral sclerosis. Eur J Neurol. https://doi.org/10.1111/ ene. 14063

37. Li S, Ren Y, Zhu W, Yang F, Zhang X, Huang X (2016) Phosphorylated neurofilament heavy chain levels in paired plasma and CSF of amyotrophic lateral sclerosis. J Neurol Sci 367:269-274. https ://doi.org/10.1016/j.jns.2016.05.062

38. Ganesalingam J, An J, Shaw CE, Shaw G, Lacomis D, Bowser R (2011) Combination of neurofilament heavy chain and complement C3 as CSF biomarkers for ALS. J Neurochem 117(3):528537. https://doi.org/10.1111/j.1471-4159.2011.07224.x 